\title{
Is Human Papillomavirus Associated with Prostate Cancer Survival?
}

\author{
Mariarosa Pascale, ${ }^{1}$ Danae Pracella, ${ }^{2}$ Renzo Barbazza, ${ }^{2}$ Barbara Marongiu, ${ }^{1}$ \\ Enrico Roggero, ${ }^{1}$ Serena Bonin, ${ }^{2}$ and Giorgio Stanta ${ }^{2}$ \\ ${ }^{1}$ Oncology Institute of Southern Switzerland (IOSI), Ospedale San Giovanni, 6500 Bellinzona, Switzerland \\ ${ }^{2}$ Department of Medical Sciences, University of Trieste, Cattinara Hospital, I-34149 Trieste, Italy
}

Correspondence should be addressed to Enrico Roggero; enrico.roggero@eoc.ch

Received 24 June 2013; Revised 10 September 2013; Accepted 7 October 2013

Academic Editor: Ahmed O. Kaseb

Copyright (c) 2013 Mariarosa Pascale et al. This is an open access article distributed under the Creative Commons Attribution License, which permits unrestricted use, distribution, and reproduction in any medium, provided the original work is properly cited.

\begin{abstract}
The role of human papillomavirus (HPV) in prostate carcinogenesis is highly controversial: some studies suggest a positive association between HPV infection and an increased risk of prostate cancer $(\mathrm{PCa})$, whereas others do not reveal any correlation. In this study, we investigated the prognostic impact of HPV infection on survival in 150 primary PCa patients. One hundred twelve (74.67\%) patients had positive expression of HPV E7 protein, which was evaluated in tumour tissue by immunohistochemistry. DNA analysis on a subset of cases confirmed HPV infection and revealed the presence of genotype 16. In Kaplan-Meier analysis, HPV-positive cancer patients showed worse overall survival (OS) (median 4.59 years) compared to HPV-negative (median 8.24 years, $P=0.0381)$. In multivariate analysis age $(P<0.001)$, Gleason score $(P<0.001)$, nuclear grading $(P=0.002)$, and HPV status $(P=0.034)$ were independent prognostic factors for OS. In our cohort, we observed high prevalence of HPV nuclear E7 oncoprotein and an association between HPV infection and PCa survival. In the debate about the oncogenic activity of HPV in $\mathrm{PCa}$, our results further confirm the need for additional studies to clarify the possible role of HPV in prostate carcinogenesis.
\end{abstract}

\section{Introduction}

Human papillomaviruses (HPVs) are small epitheliotropic viruses belonging to the Papillomaviridae family and consist of $8 \mathrm{~kb}$ double stranded circular DNA surrounded by a nonenveloped capsid. The involvement of HPV infection in a malignant transformation was first discovered by zur Hausen, who identified a subgroup of HPVs as etiological agents of cervical cancer [1-3]. More than 120 types of HPV have been identified and about one-third of them infect the genital tract $[4,5]$. These sexually transmitted viruses are classified either as "low-risk," or nononcogenic HPV-type (e.g., HPV-6 and -11), or as "high-risk," or oncogenic (e.g., HPV-16 and HPV$18)[4,5]$, and recently they have also been associated with nongenital cancers [6], particularly head and neck tumours $[7,8]$. HPV E6 and E7 are the main transforming viral proteins that work together to immortalize infected cells $[9$, 10]. E7 oncoprotein targets the retinoblastoma protein (pRB) for degradation causing a release of E2F transcription factor and the constitutive expression of E2F-responsive genes with premature activation of S-phase. Normally, this condition leads to p53-dependent apoptosis, but HPV has evolved to inhibit the tumour suppressor p53 functions, indirectly by inducing its ubiquitylation and proteasomal degradation or directly by binding p53 interfering with its transcriptional activity. Moreover, HPV oncoproteins contribute to the progressive expansion of tumours by stimulating proangiogenic cytokines, especially VEGF (vascular endothelial growth factor), responsible for the recruitment of new blood vessel capillaries from preexisting mature vessels [11-13]. Besides those, several other E7 and E6 transforming activities (reviewed in $[9,10])$, probably assisted by HPV E5, contribute to maintaining a high proliferative and inhibited apoptosis state that leads to the accumulation of mutations and genomic instability in persistently infected cells, which results in full malignant progression and cancer development. 
To date several studies have investigated the role of HPV in prostate carcinogenesis with very contradictory and not fully conclusive results. In the most recent literature, some studies suggest a positive association between HPV infection and prostate cancer risk [14-17], whereas others do not reveal any correlation [18-24]. In this study, we investigated the prevalence of HPV infection and the prognostic impact for overall survival in a cohort of patients with primary prostate cancer.

\section{Material and Methods}

2.1. Patients. Detailed histopathological and clinical data were retrospectively collected for 150 patients, who were diagnosed with primary prostate cancer in a single institution of the northeastern area of Italy from January 1992 to December 1994. Inclusion criteria were (i) the diagnosis of prostate cancer and (ii) the availability of formalin-fixed and paraffin-embedded tissues for immunohistochemical staining and molecular analyses. No fine needle biopsies were used for this study, and consequently patients with solely fine needle biopsies were excluded. In detail, following the abovementioned criteria, cases refer to patients diagnosed in the University Hospital of Trieste from 1 January 1992 to 31 December 1994. Prognostic variables were grouped for statistical analysis as reported in Table 1 . The use of formalinfixed and paraffin-embedded prostate cancer tissues and the related clinical information were approved by the Ethical Committee of the University of Trieste (Report 23; 5.10.2009) before the beginning of the study.

2.2. Tissue Microarray. Each patient's haematoxylin \& eosin (H\&E) slides were reviewed by an expert pathologist (RB), who marked the representative tumour areas to be analysed. Tissue microarrays (TMAs) were constructed by the use of the entire cohort's FFPE tissues using Galileo TMA CK3500 (Integrated Systems Engineering, Milano, Italy), as previously described [25]. Multiple tissue cores were sampled for tumours showing histological heterogeneity. One section from each TMA block was stained with H\&E to confirm the presence of carcinoma.

2.3. Immunohistochemical Staining. Immunohistochemical staining (IHC) was performed on $4 \mu \mathrm{m}$ thick tissue sections of the TMA block following the standard procedures [26]. To detect HPV E7 protein, Cervimax IHC kit (Valdospan $\mathrm{GmbH}$, Austria) was used as already reported [27]. Immunostaining was performed manually with the Vectastain Universal Elite ABC kit (Vector Laboratories, Burlingame, CA, USA). In short, after deparaffinisation, alcohol washings, and endogenous peroxidase blocking, TMAs were treated with 3\% BSA for aspecific sites blocking and subsequently incubated with E7 mAb (1:200 dilution) for 1 hour at room temperature. For the visualization of reaction DAB Substrate kit for Peroxidase (Vector Laboratories, Burlingame, CA, USA) was used. Positive and negative control slides were used in each IHC assay. For evaluation of the immunostaining, the positively stained cells were counted across 3 high-power fields at 40x magnification; cytoplasmatic and nuclear staining were recorded separately. For IHC, the specificity of HPV E7 signal was assessed with a preabsorption test performed on a prostate cancer. Two aliquots of the working dilutions of mAb E7 were used: one was mixed with the immunizing peptide (kindly provided by Valdospan $\mathrm{GmbH}$, Austria) in a stoichiometrical ratio of $1: 5$ and the other was used undiluted. Both were incubated with the specimen for 30 minutes at $37^{\circ} \mathrm{C}$ before IHC staining.

2.4. PCR and Sequencing. Twenty-two cancer cases, 19 positive and 3 negative for HPV E7 immunodetection, were submitted to DNA extraction using a homemade protocol [28]. In short, dewaxed tissues sections were incubated overnight at $55^{\circ} \mathrm{C}$ with proteinase $\mathrm{K}$. Crude extracts were purified by phenol/chloroform and concentrated by ethanol precipitation. To check the availability and quality of the DNA, extracts were submitted to multiplex PCR using the specimens control size DNA ladder (In Vivo Scribe Technologies), which enables amplifying 100, 200, 300, 400, and $600 \mathrm{bp}$ DNA fragments [29]. DNA extracts were tested for the presence and type of HPV with a PCR-based system by using the GP5+/6+ consensus primers set $\left(5^{\prime}\right.$-TTTGTTACTGTGGTAGATACTAC- $3^{\prime}$ and $5^{\prime}$-GAAAAATAAACTGTAAATCATATTC- $3^{\prime}$ ), which targets conserved sequences in the $\mathrm{L} 1$ gene and enables amplifying a wide spectrum of HPV types [30]. PCR was run for 45 cycles, and then products were run on a $2 \%$ Metaphor agarose gel and 14 samples were purified by the use of QIAquick PCR purification kit (Qiagen) following the manufacturer's instruction [31]. After this step, purified amplicons were submitted to Sanger sequencing for genotyping (BMR Genomics, Padua, Italy). Reamplification of purified PCR products and nested PCR were avoided because of the higher probability of PCR contamination.

2.5. Statistical Analysis. Overall survival (OS), defined as the time between the date of diagnosis and the date of death or the last follow-up (FU) observation, was the end point evaluated in this study. Patients were censored if they were still alive or they were lost to FU. The Kaplan-Meier method was used to generate cancer-specific survival curves and differences between groups were analysed using the log-rank test. A multivariate Cox proportional hazards analysis was used to evaluate the association of OS with each prognostic factor. A $\chi^{2}$ test was applied to determine whether age at diagnosis was equally distributed between dichotomized groups for each variable used for OS analysis. Possible associations among covariates were evaluated by $\chi^{2}$ test. All statistical tests were done by STATA software (StataCorp. 2011. Stata Statistical Software: Release 12. College Station, TX: StataCorp LP) and a value of $P \leq 0.05$ was considered statistically significant.

\section{Results}

3.1. Patients' Characteristics. Table 1 summarizes the baseline characteristics of the 150 patients included in the study. The median age at diagnosis was 72.18 years (range: 50.57 to 91.13 years) and the median FU time was 5 years (range: 0.1 to 18.85 years). Nuclear E7 staining, which is a marker of HPV 
TABLE 1: Univariate and multivariate analyses of prognostic factors in 150 patients with primary prostate cancer. Univariate analysis was performed by using the Kaplan-Meier method. Multivariate analysis was done by the Cox proportional hazards model.

\begin{tabular}{|c|c|c|c|c|c|c|c|c|c|}
\hline \multirow[b]{2}{*}{ Variable } & \multirow{2}{*}{$\begin{array}{c}\text { Patients } \\
n=150(\%)\end{array}$} & \multirow{2}{*}{$\begin{array}{c}\text { Deaths } \\
n=132(\%)\end{array}$} & \multicolumn{3}{|c|}{ Univariate } & \multicolumn{4}{|c|}{ Multivariate } \\
\hline & & & $\begin{array}{l}\text { Median survival } \\
\text { time (years) }\end{array}$ & $95 \% \mathrm{CI}$ & $P$ value & Hazard ratio & Standard error & $95 \% \mathrm{CI}$ & $P$ value \\
\hline \multicolumn{10}{|l|}{ Age (years) } \\
\hline$\leq 72$ & 85 (56.67) & $69(52.27)$ & 6.87 & $5.15-9.29$ & \multirow{2}{*}{$<0.001^{*}$} & \multirow{2}{*}{2.15} & \multirow{2}{*}{0.39} & \multirow{2}{*}{$1.51-3.06$} & \multirow{2}{*}{$<0.001^{*}$} \\
\hline$>72$ & $65(43.33)$ & $63(47.73)$ & 3.14 & $1.82-3.63$ & & & & & \\
\hline \multicolumn{10}{|c|}{ Nuclear grade } \\
\hline 1 & $23(15.33)$ & $18(13.64)$ & 13.60 & $6.21-15.13$ & \multirow{3}{*}{$<0.001^{*}$} & & & & \\
\hline 2 & $99(66.00)$ & $86(65.15)$ & 5.14 & $4.12-6.73$ & & 1.53 & 0.42 & $0.91-2.62$ & 0.105 \\
\hline 3 & $28(18.67)$ & $28(21.21)$ & 2.56 & $1.34-3.63$ & & 2.76 & 0.92 & $1.43-5.31$ & $0.002^{*}$ \\
\hline \multicolumn{10}{|c|}{ Capsule infiltration } \\
\hline Negative & $84(56.37)$ & $71(54.20)$ & 6.21 & $4.72-8.79$ & \multirow{3}{*}{$0.0159^{*}$} & & & & \\
\hline Positive & $65(43.63)$ & $60(45.80)$ & 3.30 & $1.89-4.95$ & & & & & \\
\hline 1 missing & & & & & & & & & \\
\hline \multicolumn{10}{|c|}{ Gleason score } \\
\hline$<7$ & $64(42.67)$ & $49(37.12)$ & 10.78 & $6.91-13.60$ & \multirow{2}{*}{$<0.001^{*}$} & \multirow{2}{*}{2.53} & \multirow{2}{*}{0.51} & \multirow{2}{*}{$1.70-3.75$} & \multirow{2}{*}{$<0.001^{*}$} \\
\hline$\geq 7$ & $86(57.33)$ & $83(62.88)$ & 3.28 & $1.85-4.59$ & & & & & \\
\hline \multicolumn{10}{|l|}{ Nuclear } \\
\hline \multicolumn{10}{|c|}{ E7 expression } \\
\hline Negative & $38(25.33)$ & $30(22.73)$ & 8.24 & $3.63-11.81$ & \multirow{2}{*}{$0.0381^{*}$} & \multirow{2}{*}{1.57} & \multirow{2}{*}{0.33} & \multirow{2}{*}{$1.03-2.39$} & \multirow{2}{*}{$0.034^{*}$} \\
\hline Positive & $112(74.67)$ & $102(77.27)$ & 4.59 & $3.45-5.35$ & & & & & \\
\hline
\end{tabular}

CI: confidence interval; ${ }^{*}$ significant value.

infection [9], was positive in $112(74.67 \%)$ patients at the time of diagnosis. One hundred thirty-two (88\%) patients died at the end of the observation period and those patients who were still alive were censored for survival analyses. Considering the elevated median age at diagnosis and the high mortality rate of our cohort, we performed a $\chi^{2}$ test that demonstrated that there were no significant differences in distribution of age at diagnosis in the nuclear grade $\left(\chi^{2}=\right.$ 1.51 , degree of freedom $(\mathrm{df})=2, P=0.47)$, capsule infiltration $\left(\chi^{2}=0.046, \mathrm{df}=1, P=0.83\right)$, Gleason score $\left(\chi^{2}=3.64\right.$, $\mathrm{df}=1, P=0.06)$, and nuclear E7 expression $\left(\chi^{2}=0.87\right.$, $\mathrm{df}=1, P=0.35$ ) groups for OS analysis.

3.2. HPV Detection. The specificity of the E7 mAb signal detected by IHC was assessed by the pre-absorption test. As reported in Figure 1, the staining pattern was completely eliminated after incubation of the antibody with the immunizing peptide. Both cytoplasmatic and nuclear staining were evaluated. Diffuse or granular immunostaining was observed. In some cases, a combination of both was detected. Cells characterised by a diffuse or focal staining pattern were considered positive. A representative staining of E7 is reported in Figure 2. HPV E7 positivity prevailed at the nuclear level with intensity varying from 1 to 3 . Although nuclear immunostaining was the most represented one, cases presenting with cytoplasmatic positivity were also observed (data not shown).
3.3. PCR Analyses. Specimen control size was successful in all prostate cancer tissues; in detail in 19 out of 22 samples 300 bp fragments were detectable and in 3 out of 22 cases 200 bases fragments were visualized. HPV PCR amplification resulted positively in 18 out of 19 specimens positive for HPV E7 immunodetection with variable intensities (Figure 1(c)). Variability and failure of PCR amplification in some samples could be due to the low DNA quality in some formalin fixed-paraffin embedded tissues. It is well known, indeed, that degradation of nucleic acids represents a limit in the detection of HPV by extractive methods in formalin fixed-paraffin embedded biopsies [32, 33]. All three cases negative for HPV E7 immunodetection were confirmed negative by PCR. All purified amplification products were submitted to sequencing; in 9 out of 14 specimens sequencing was successful and showed that in those cases the HPV genotype was 16; in the remaining ones the sequence was not readable because of the inadequate amount of PCR products submitted to Sanger reaction (data not shown). Typical results of sequencing were as follows: ATGTGCTGCATATCTACTTCAGAAACTACATATAAAAATACTAACTTTAAGGAGTACCTACGACATGGGGAGGAATATGATTTACAGTTTATTTTTCA, which gave $98-99 \%$ of identity with human papillomavirus type 16 .

3.4. Univariate Analysis. Association of each factor with survival time was calculated by the Kaplan-Meier method and survival differences were evaluated by the log-rank test. 


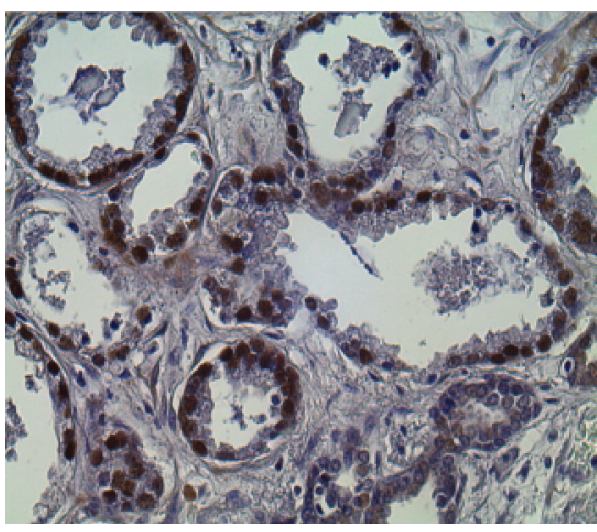

(a)

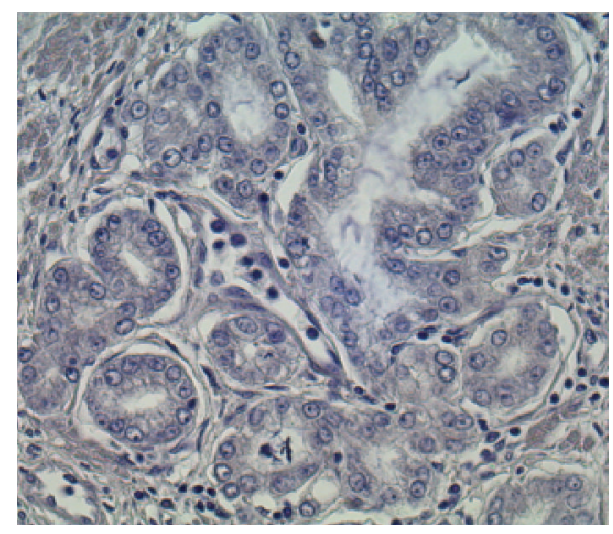

(b)
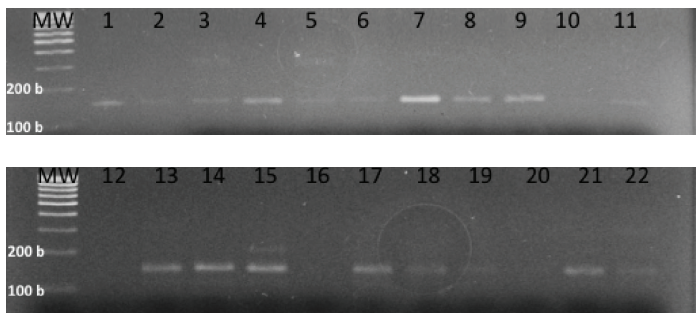

(c)

Figure 1: Tests for specificity of the analysis. Preabsorption test to verify the specificity of the E7 signal on prostate cancer samples. Immunostaining with the Cervimax IHC kit in prostate cancer tissue (a) and after preabsorption with the specific oncoviral peptide (b). Original magnification: 20x; conventional immunohistochemistry performed with diaminobenzidine as a chromogen and hematoxylin for counterstaining. PCR amplification of HPV DNA is reported in (c): MW molecular weight marker, 1-22 amplification carried out in prostate cancer specimens. In 19 cases positive for HPV E7 IHC, 18 were PCR positive and 1 was PCR negative (sample 10). Of those successfully sequenced samples were 4, 7, 8, 9, 13,14, 15, 17, and 21. Sequencing failed in samples 1, 2, 3, 5, and 6. Samples 12, 16, and 20 refer to amplification carried out in prostate cancer cases negative for HPV E7 immunodetection.

Univariate survival analyses showed that the age at diagnosis $(P<0.001)$, Gleason score $(P<0.001)$, nuclear grade $(P<0.001)$, capsule infiltration $(P=0.0159)$, and nuclear E7 expression $(P=0.0381)$ were strongly related to OS (Table 1). Interestingly, HPV-positive cancer patients showed worse OS (median 4.59 years) compared to those who were HPV negative (median 8.24 years, $P=0.0381$; Figure 3 ). Thus, these results demonstrate that HPV infection significantly affects survival time of prostate cancer patients in our cohort.

3.5. Multivariate Analysis. Prognostic factors associated with survival time in the univariate analysis were evaluated in a Cox multivariate regression model. Capsule infiltration was eliminated from the multivariate model because of association with Gleason score $\left(\chi^{2}=13.28\right.$, df $=1, P<$ $0.001)$ and nuclear grade $\left(\chi^{2}=11.29\right.$, $\mathrm{df}=2, P=$ $0.004)$. In this analysis the age at diagnosis, Gleason score, nuclear grade, and HPV status were statistically independent predictors for OS with, respectively, HRs of 2.15 (95\% CI, 1.513.06; $P<0.001$ ), 2.53 (95\% CI, 1.70-3.75; $P<0.001$ ), 2.76 (95\% CI, 1.43-5.31; $P=0.002$ ), and 1.57 (95\% CI, 1.03-2.39; $P=0.034)$ (Table 1).

\section{Discussion}

Current literature on the role of HPV infection in the carcinogenesis of prostate cancer remains controversial. Recently, Martinez-Fierro et al. [16] evaluated the presence of viral HPV DNA in 55 prostatic cancer tissues and 75 controls and found a significant positive association between HPV and risk of prostate cancer. In the same year, Sutcliffe et al. [18] did not find any association in a prospective study on serum samples collected from 612 patients with prostate cancer and 612 controls. No positive correlation has been reported in subsequent serologic [22] or viral DNA-based $[19,21,23]$ or mixed laboratory technique $[20,24]$ studies. More recently, Whitaker et al. [34] observed a ubiquitous distribution of HPV in normal, benign, and cancerous prostate tissues supporting the innocuity of HPV-18; however, they concluded an oncogenic potential role of HPV-18 in prostate cancer, since they detected HPV-18 in koilocytes of prostatic tissues. Even about the most representative HPV types, there is great discordance. If Whitaker and colleagues reported the potential involvement of HPV-18 in prostate cancer [34], Lin et al. [17] found a prevalence of HPV-16 with respect to HPV18 in a meta-analysis, whereas Adami et al. [14] observed a 


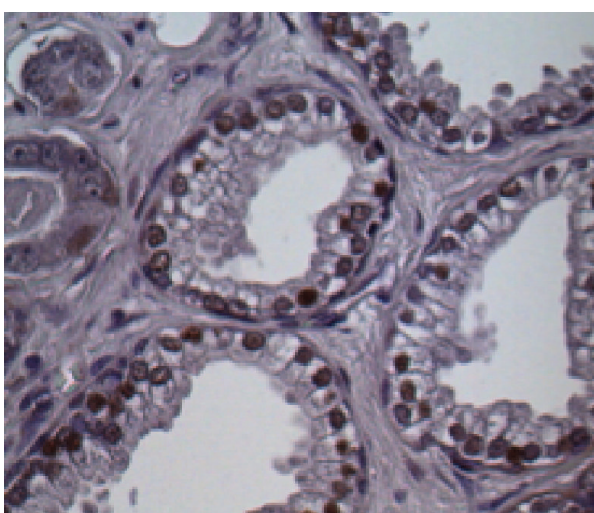

(a)

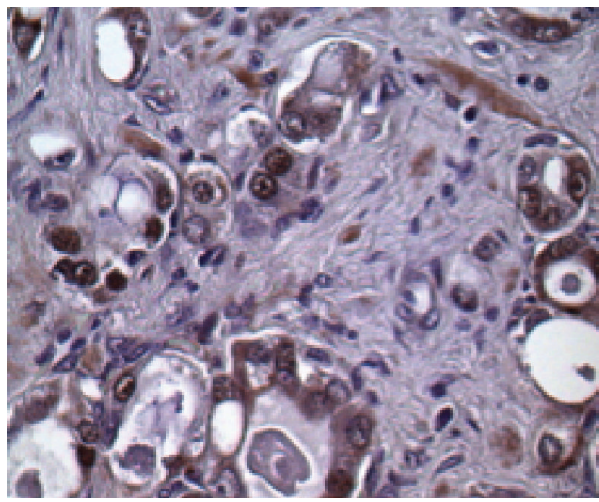

(c)

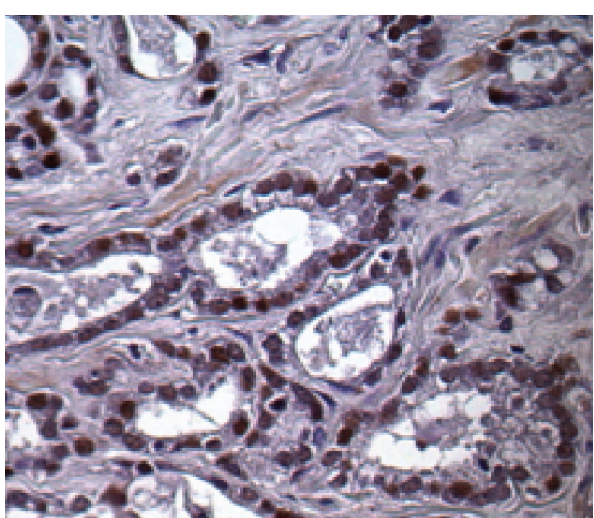

(b)

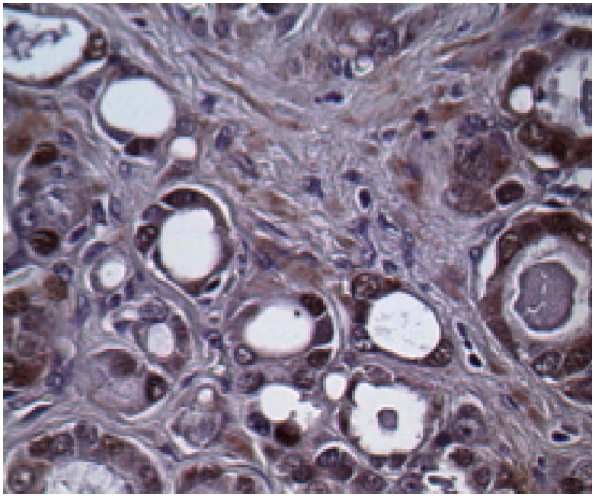

(d)

FIGURE 2: Representative immunohistochemistry staining of E7 for prostate intraepithelial neoplasia (PIN2) (a); prostate adenocarcinomas (b), (c), and (d); original magnification: 20x; conventional immunohistochemistry performed with diaminobenzidine as a chromogen and hematoxylin for counterstaining.

positive association between prostate cancer and HPV-33 and no oncogenic role for HPV-16 and -18. A major prevalence of HPV-16 has been reported by Leiros and colleagues [15]. Therefore, these mixed results are consistent with the present controversial landscape on the role of HPV infection in prostate carcinogenesis.

In this study, we investigated the clinical significance of HPV infection in primary prostate cancer patients. We found that overall survival was significantly associated with the nuclear expression of E7 protein and other clinicalpathological factors, such as age higher than 72 years, high nuclear grade, capsule infiltration, and high Gleason score at diagnosis. The statistical independence of each of these prognostic factors was assessed in a multivariate analysis, which identified age, nuclear grade, Gleason score, and HPV E7 expression as significantly independent prognostic factors for survival of patients with primary prostate cancer. Furthermore, analysis of DNA extracted from all the analysed samples of our cohort confirmed HPV infection and showed the presence of HPV16 genotype. This result is consistent with other studies on prostate cancer $[15,17]$ and further reinforces the high carcinogenicity of HPV16, which has already been shown as the most carcinogenic HPV genotype for cervical $[6,35]$ and neck and head cancers [6]. Although our data do not provide supportive proof for a causal relation between
HPV and prostate cancer, they demonstrate a potential association between HPV infection and adverse prognosis in our patient cohort. Moreover, they sustain a possible role of $\mathrm{HPV}$ as an independent prognostic factor for OS of primary prostate cancer patients. We believe that our data, which analyse the prognostic impact of HPV infection in prostate cancer from a clinical point of view, confirm the absolute need to investigate and validate the prevalence of HPV infection in a controlled prospective study and its role in prostate carcinogenesis. This study is increasingly important as we have $\mathrm{HPV}$ vaccines able to guarantee excellent prevention and control of this viral infection and its consequences in terms of tumour development. Moreover, the correlation between HPV infection and angiogenesis [11-13] offers a good rationale to treat a specific subgroup of prostate cancer patients selected on the basis of the HPV status by using antiangiogenic agents, whose efficacy has recently been reported for the treatment of advanced cervical cancer [36].

\section{Conclusion}

This study provides evidence for the correlation between $\mathrm{HPV}$ infection and prostate cancer survival in our patient cohort and sustains the need for additional studies to clarify the possible role of HPV in prostate carcinogenesis. Further 


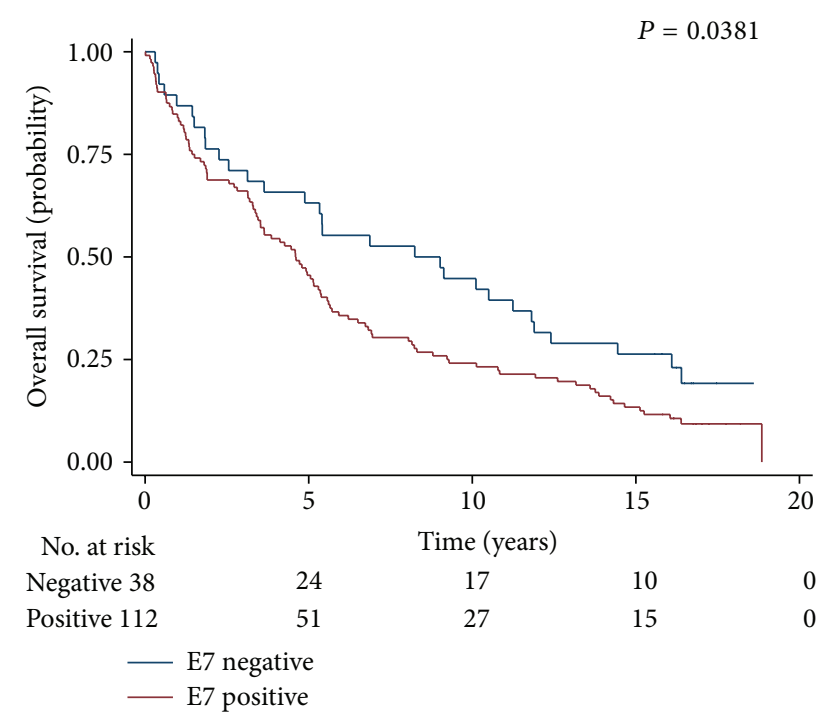

FIgURE 3: Survival curves by HPV E7 nuclear expression in 150 patients with primary prostate cancer. $P$ value from log-rank test is reported. Numbers of at risk (still alive) patients are indicated below the $x$-axis.

studies are required because vaccines and novel therapeutic drugs are now available and may be used to prevent HPV infection and treat more effectively HPV-associated prostate cancer.

\section{Conflict of Interests}

The authors declare that they have no conflict of interests.

\section{Authors' Contribution}

Mariarosa Pascale and Danae Pracella contributed equally to the realisation of this work.

\section{Acknowledgments}

This work was supported by grants from ABREOC 2011. Mariarosa Pascale was supported by an ESO fellowship. The authors would like to thank Dr. Valentina Melita for the language revision of the paper.

\section{References}

[1] H. zur Hausen, "Human papillomaviruses and their possible role in squamous cell carcinomas," Current Topics in Microbiology and Immunology, vol. 78, pp. 1-30, 1977.

[2] M. Dürst, L. Gissmann, H. Ikenberg, and H. zur Hausen, "A papillomavirus DNA from a cervical carcinoma and its prevalence in cancer biopsy samples from different geographic regions," Proceedings of the National Academy of Sciences of the United States of America, vol. 80, no. 12 I, pp. 3812-3815, 1983.

[3] M. Boshart, L. Gissmann, H. Ikenberg, A. Kleinheinz, W. Scheurlen, and H. zur Hausen, "A new type of papillomavirus DNA, its presence in genital cancer biopsies and in cell lines derived from cervical cancer," The EMBO Journal, vol. 3, no. 5, pp. 1151-1157, 1984.

[4] E. M. de Villiers, C. Fauquet, T. R. Broker, H. U. Bernard, and H. zur Hausen, "Classification of papillomaviruses," Virology, vol. 324, no. 1, pp. 17-27, 2004.

[5] H. U. Bernard, R. D. Burk, Z. Chen, K. van Doorslaer, H. Z. Hausen, and E. M. de Villiers, "Classification of papillomaviruses (PVs) based on 189 PV types and proposal of taxonomic amendments," Virology, vol. 401, no. 1, pp. 70-79, 2010.

[6] IARC Working Group on the Evaluation of Carcinogenic Risks to Humans, "Human papillomaviruses", in IARC Monographs on the Evaluation of Carcinogenic Risks To Humans. A Review of Human Carcinogens, International Agency for Research on Cancer, Ed., vol. 100, part B of Biological Agents, pp. 255-314, WHO, Geneva, Switzerland, 2012.

[7] M. L. Gillison, W. M. Koch, R. B. Capone et al., "Evidence for a causal association between human papillomavirus and a subset of head and neck cancers," Journal of the National Cancer Institute, vol. 92, no. 9, pp. 709-720, 2000.

[8] A. Argiris, M. V. Karamouzis, D. Raben, and R. L. Ferris, "Head and neck cancer," The Lancet, vol. 371, no. 9625, pp. 1695-1709, 2008.

[9] C. A. Moody and L. A. Laimins, "Human papillomavirus oncoproteins: pathways to transformation," Nature Reviews Cancer, vol. 10, no. 8, pp. 550-560, 2010.

[10] M. E. McLaughlin-Drubin, J. Meyers, and K. Munger, "Cancer associated human papillomaviruses," Current Opinion in Virology, vol. 2, pp. 459-466, 2012.

[11] O. López-Ocejo, A. Viloria-Petit, M. Bequet-Romero, D. Mukhopadhyay, J. Rak, and R. S. Kerbel, "Oncogenes and tumor angiogenesis: the HPV-16 E6 oncoprotein activates the vascular endothelial growth factor (VEGF) gene promoter in a p53 independent manner," Oncogene, vol. 19, no. 40, pp. 4611-4620, 2000.

[12] L. Xi, S. Wang, C. Wang et al., "The pro-angiogenic factors stimulated by human papillomavirus type 16 E6 and E7 protein in C33A and human fibroblasts," Oncology Reports, vol. 21, no. 1, pp. 25-31, 2009.

[13] B. M. El Sabaa, M. Meleiss, and I. Zaki, "EGF expression and microvascular density in relation to highrisk-HPV infection in cervical carcinoma-an immunohistochemical study," The American Journal of Medicine, vol. 48, pp. 47-57, 2012.

[14] H. O. Adami, H. Kuper, S. O. Andersson, R. Bergström, and J. Dillner, "Prostate cancer risk and serologic evidence of human papilloma virus infection: a population-based case-control study," Cancer Epidemiology Biomarkers and Prevention, vol. 12, no. 9, pp. 872-875, 2003.

[15] G. J. Leiros, S. R. Galliano, M. E. Sember, T. Kahn, E. Schwarz, and K. Eiguchi, "Detection of human papillomavirus DNA and p53 codon 72 polymorphism in prostate carcinomas of patients from Argentina," BMC Urology, vol. 5, article 15, 2005.

[16] M. L. Martinez-Fierro, R. J. Leach, L. S. Gomez-Guerra et al., "Identification of viral infections in the prostate and evaluation of their association with cancer," BMC Cancer, vol. 10, article 326, 2010.

[17] Y. Lin, Q. Mao, X. Zheng et al., "Human papillomavirus 16 or 18 infection and prostate cancer risk: a meta-analysis," Irish Journal of Medical Science, vol. 180, no. 2, pp. 497-503, 2011.

[18] S. Sutcliffe, R. P. Viscidi, C. Till et al., "Human papillomavirus types 16,18 , and 31 serostatus and prostate cancer risk in 
the prostate cancer prevention trial," Cancer Epidemiology Biomarkers and Prevention, vol. 19, no. 2, pp. 614-618, 2010.

[19] A. Aghakhani, R. Hamkar, M. Parvin et al., "The role of human papillomavirus infection in prostate carcinoma," Scandinavian Journal of Infectious Diseases, vol. 43, no. 1, pp. 64-69, 2010.

[20] A. C. Chen, T. Waterboer, A. Keleher et al., "Human papillomavirus in benign prostatic hyperplasia and prostatic adenocarcinoma patients," Pathology and Oncology Research, vol. 17, no. 3, pp. 613-617, 2011.

[21] H. C. T. Groom, A. Y. Warren, D. E. Neal, and K. N. Bishop, "No evidence for infection of UK prostate cancer patients with $\mathrm{XMRV}, \mathrm{BK}$ virus, trichomonas vaginalis or human papilloma viruses," PLoS ONE, vol. 7, no. 3, Article ID e34221, 2012.

[22] J. Hrbacek, M. Urban, E. Hamsikova et al., "Serum antibodies against genitourinary infectious agents in prostate cancer and benign prostate hyperplasia patients: a case-control study," $B M C$ Cancer, vol. 11, article 53, 2011.

[23] A. Rogler, M. Rogenhofer, A. Borchardt et al., "P53 codon 72 (Arg72Pro) polymorphism and prostate cancer risk: association between disease onset and proline genotype," Pathobiology, vol. 78, no. 4, pp. 193-200, 2011.

[24] R. Tachezy, J. Hrbacek, J. Heracek et al., "HPV persistence and its oncogenic role in prostate tumors," Journal of Medical Virology, vol. 84, pp. 1636-1645, 2012.

[25] V. Faoro and A. Sapino, “Tissue microarray (TMA)," in Guidelines for Molecular Analysis in Archive Tissues, G. Stanta, Ed., pp. 23-26, Springer, Berlin, Germany, 2011.

[26] N. S. Goldstein, S. M. Hewitt, C. R. Taylor et al., "Recommendations for improved standardization of immunohistochemistry," Applied Immunohistochemistry \& Molecular Morphology, vol. 15, no. 2, pp. 124-133, 2007.

[27] V. Faoro, R. Barbazza, S. Bonin, D. Brunetti, S. Sulfaro, and G. Stanta, "Detection of HPV E7 oncoviral protein in cervical lesions by a new antibody," Applied Immunohistochemistry \& Molecular Morphology, vol. 21, pp. 341-350, 2013.

[28] P. Pauluzzi, S. Bonin, M. A. Gonzalez Inchaurraga, G. Stanta, and G. Trevisan, "Detection of spirochaetal DNA simultaneously in skin biopsies, peripheral blood and urine from patients with erythema migrans," Acta Dermato-Venereologica, vol. 84, no. 2, pp. 106-110, 2004.

[29] J. J. M. van Dongen, A. W. Langerak, M. Brüggemann et al., "Design and standardization of PCR primers and protocols for detection of clonal immunoglobulin and T-cell receptor gene recombinations in suspect lymphoproliferations: report of the BIOMED-2 concerted action BMH4-CT98-3936," Leukemia, vol. 17, no. 12, pp. 2257-2317, 2003.

[30] I. Petersen, C. Schewe, K. Schlüns et al., "Inter-laboratory validation of PCR-based HPV detection in pathology specimens," Virchows Archiv, vol. 451, no. 3, pp. 701-716, 2007.

[31] G. Basili, S. Bonin, and I. Dotti, "DNA sequencing from PCR products," in Guidelines for Molecular Analysis in Archive Tissues, G. Stanta, Ed., pp. 135-141, Springer, Berlin, Germany, 2011.

[32] K. Biedermann, N. Dandachi, M. Trattner et al., "Comparison of real-time PCR signal-amplified in situ hybridization and conventional PCR for detection and quantification of human papillomavirus in archival cervical cancer tissue," Journal of Clinical Microbiology, vol. 42, no. 8, pp. 3758-3765, 2004.

[33] M. Steinau, S. S. Patel, and E. R. Unger, "Efficient DNA extraction for HPV genotyping in formalin-fixed, paraffin-embedded tissues," Journal of Molecular Diagnostics, vol. 13, no. 4, pp. 377381, 2011.
[34] N. J. Whitaker, W. K. Glenn, A. Sahrudin, M. M. Orde, W. Delprado, and J. S. Lawson, "Human papillomavirus and Epstein Barr virus in prostate cancer: koilocytes indicate potential oncogenic influences of human papillomavirus in prostate cancer," Prostate, vol. 73, pp. 236-241, 2013.

[35] S. de Sanjose, W. G. Quint, L. Alemany et al., "Human papillomavirus genotype attribution in invasive cervical cancer: a retrospective cross-sectional worldwide study," The Lancet Oncology, vol. 11, pp. 1048-1056, 2010.

[36] S. K. Tewari, M. Sill, J. H. Long et al., "Incorporation of bevacizumab in the treatment of recurrent and metastatic cervical cancer: a phase III randomized trial of the gynecologic oncology group," Journal of Clinical Oncology, vol. 31, supplement, 2013, abstract no. 3 . 


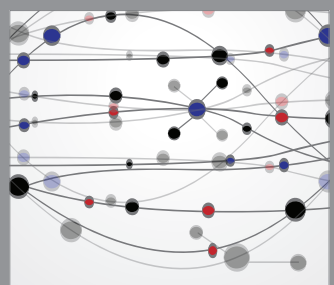

The Scientific World Journal
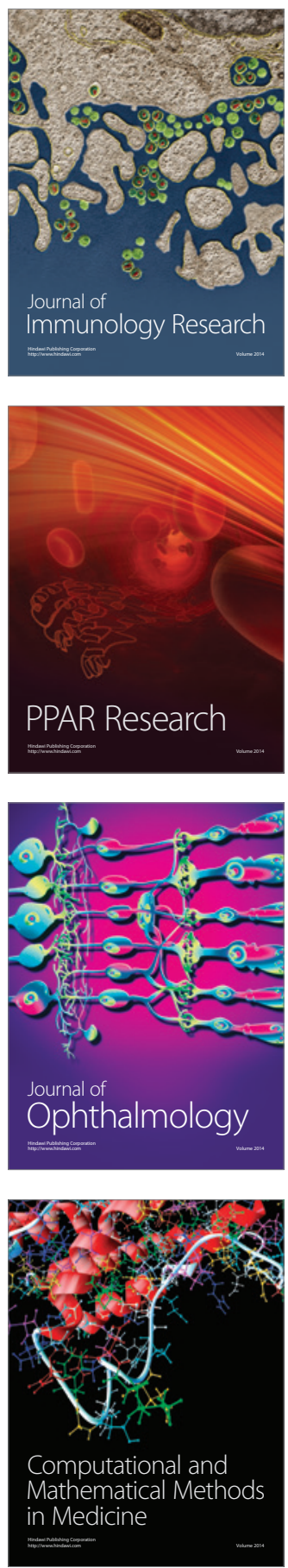

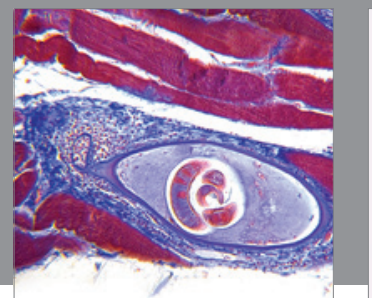

Gastroenterology

Research and Practice
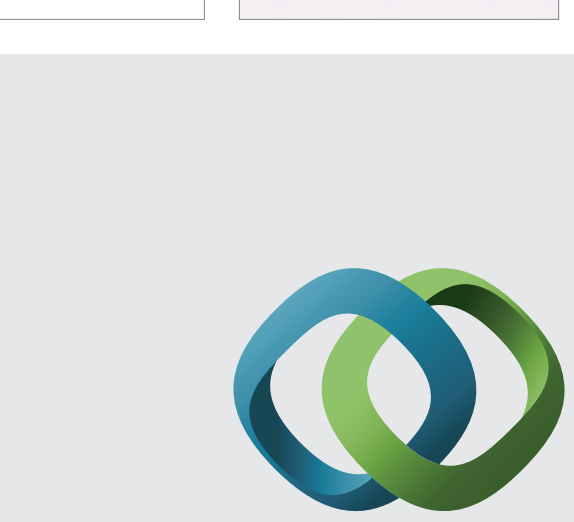

\section{Hindawi}

Submit your manuscripts at

http://www.hindawi.com
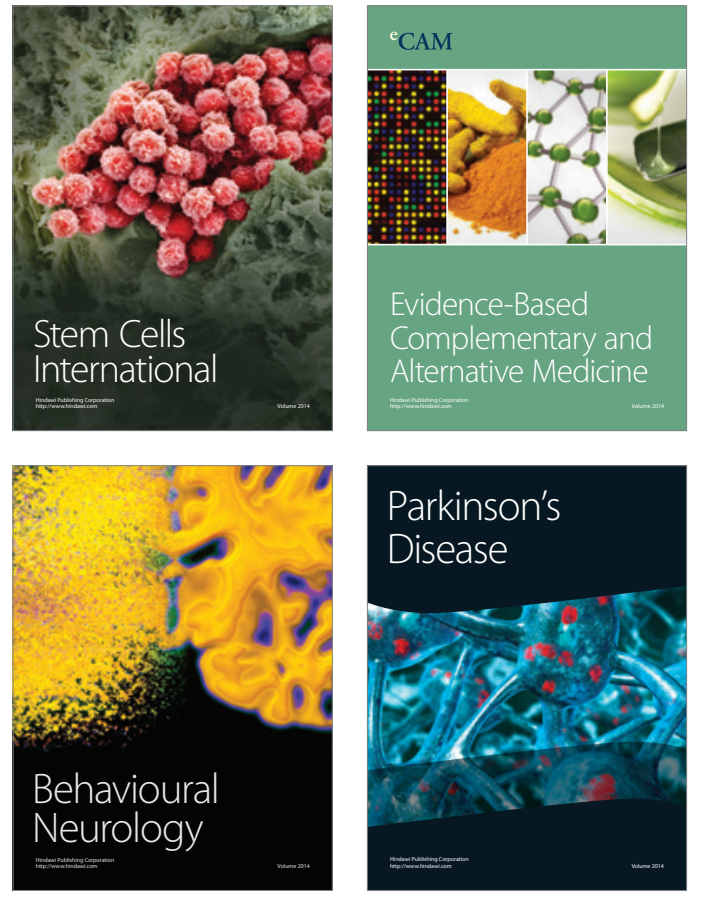
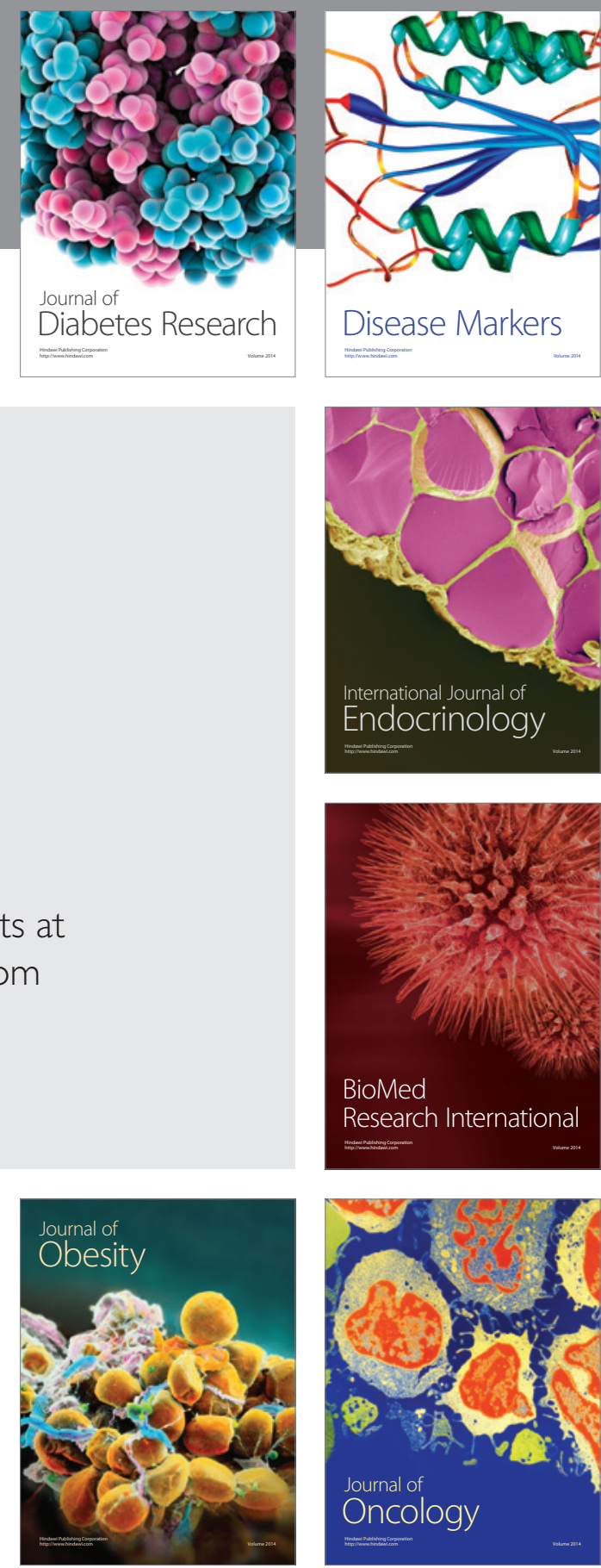

Disease Markers
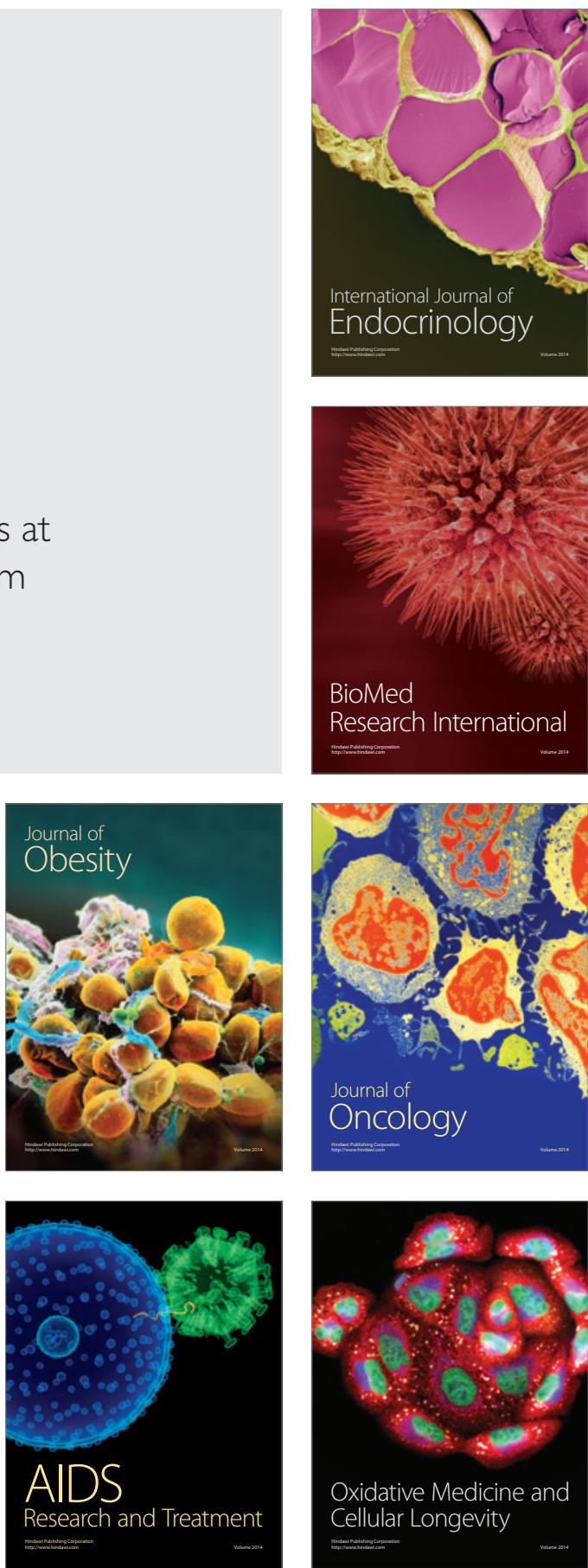